\title{
Anti-CD20/CD3 Monoclonal Antibody XmAb13676
}

National Cancer Institute

\section{Source}

National Cancer Institute. Anti-CD20/CD3 Monoclonal Antibody XmAb13676. NCI

Thesaurus. Code C130050.

\begin{abstract}
A bispecific, Fc domain-containing, monoclonal antibody with potential antineoplastic activity. Anti-CD20/CD3 monoclonal antibody XmAb13676 contains two antigenrecognition sites: one for human CD3, a T cell surface antigen, and one for human CD20, a tumor-associated antigen (TAA) that is exclusively expressed on B-cells during most stages of B-cell development and is often overexpressed in B-cell malignancies. Upon administration, XmAb13676 binds to both T-cells and CD20-expressing B-lineage tumor cells. The resulting cross-linkage may trigger a potent cytotoxic T-lymphocyte (CTL) response against the CD20-expressing tumor B-cells. Inclusion of an Fc domain on the antibody prolongs the half-life of the bispecific antibody and enhances T-cell-mediated tumor cell killing because the agent is able to bind to Fc receptors.
\end{abstract}

